\title{
Could ultrasound-guided internal jugular vein catheter insertion replace the use of chest X-ray?
}

\author{
William F. Amaya-Zuñiga ${ }^{1 *}$ (D) and Fernando Raffán-Sanabria ${ }^{2}$
}

The implementation of ultrasound protocols during catheter placement has demonstrated multiple advantages that increase accuracy and allow medical teams to reduce operative time, potential complications, and procedure costs $[1,2]$. The wide use of lung and cardiac ultrasound in perioperative medicine enables earlier diagnosis of some acute complications, allowing prompt intervention and control of damage.

We present the Successful Ultrasound-Guided Internal Jugular Vein Catheter Insertion algorithm (Fig. 1), which is based on reports of safe vascular ultrasound protocols in daily practice $[3,4]$. It follows an operational pipeline that integrates steps focused on adequate vascular identification, cannulation, and placement of the catheter. The operational steps are:

\section{1, 2 - A 2D vascular ultrasound in the Trendelenburg} position identifies vessel characteristics and determines the "margin of safety". If an overlap of the artery is present, there is a high possibility of artery injury. 3-Assessment of color Doppler and pulsed Doppler completes the vascular identification, which can differentiate their flows and discount the presence of thrombus.

4, 5 - Evaluation of a safety margin could guide modifications of puncture angles, avoiding injuries of the anterior or lateral artery wall.

6-After getting venous return, guidewire advancement should be smooth and unobstructed and the thoracic direction needs to be confirmed. The presence of detected. intraluminal dissection or false pathways should be

7, 8 - In the subcostal echocardiogram view, guidewire and catheter identification close to the heart helps to optimize adequate placement. The saline flush confirmation test can help to confirm this, but it should not be performed routinely.

9- Although the use of ultrasound in real time for central venous access reduces the number of pleuropulmonary complications, a lung ultrasound assessment should be performed, seeking to identify potential associated lesions, guaranteeing prompt attention to the patient.

The application of this algorithm optimizes the use of bedside ultrasound as a diagnostic tool, organizes the steps, and increases the early identification of potential operational errors. Rapid interventions significantly reduce the clinical impact on the patient and avoid the use of chest X-ray. Limitations for its implementation include the availability of the equipment with probes and staff training. This algorithm could also be useful in subclavian venous catheter placement, although the evidence does not statistically support the use of ultrasound for this application.

\footnotetext{
Authors' contributions

WAZ and FRS contributed substantially to the design and writing of the manuscript. WAZ was responsible for obtaining the Permission Request from the Sociedad Colombiana de Anestesiología y Reanimación S.C.A.R.E., for the modification and publication of the algorithm of the article Canalización venosa yugular interna: ¿qué tanta seguridad podemos llegar a ofrecer? (Internal jugular vein cannulation: How much safety can we offer? [3]). Both authors were responsible for review and editing of the manuscript. Both authors read and approved the final manuscript.
}

Ethics approval and consent to participate Not applicable.

\footnotetext{
* Correspondence: Williamf.amayaz@yahoo.es

${ }^{1}$ Department of Anesthesia and Perioperative Medicine, Cardiovascular and Transplant Anesthesiologist, Hospital Universitario Fundación Santa Fe de Bogotá, Universidad de los Andes, Universidad el Bosque, Cll 119 \# 7-75, Bogotá, Colombia

Full list of author information is available at the end of the article
}

\section{Consent for publication} Not applicable.

\section{Competing interests}

The authors declare that they have no competing interests. 


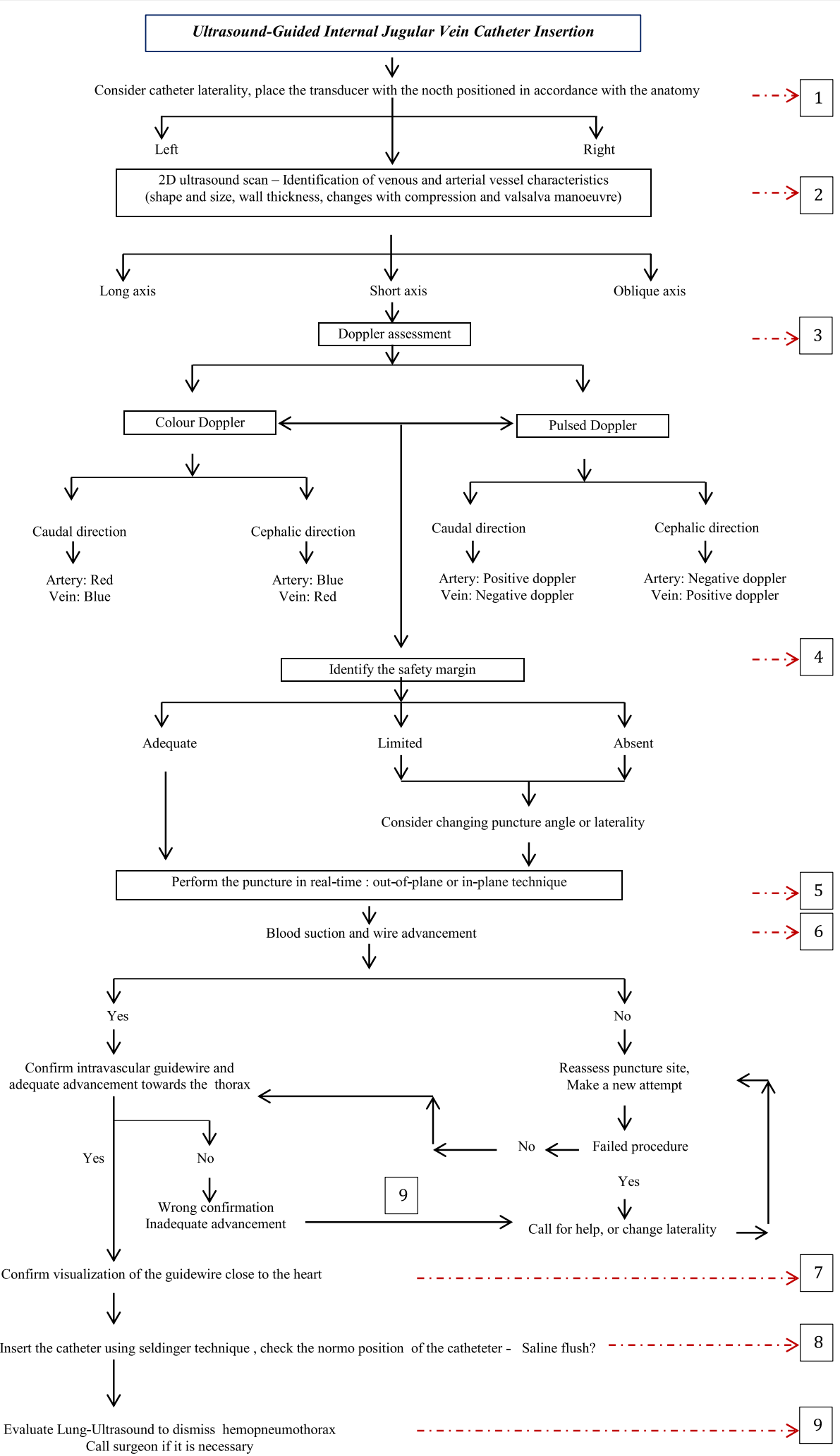

Fig. 1 Ultrasound-Guided Internal Jugular Vein Catheter Insertion algorithm 


\section{Publisher's Note}

Springer Nature remains neutral with regard to jurisdictional claims in published maps and institutional affiliations.

\section{Author details}

${ }^{1}$ Department of Anesthesia and Perioperative Medicine, Cardiovascular and Transplant Anesthesiologist, Hospital Universitario Fundación Santa Fe de Bogotá, Universidad de los Andes, Universidad el Bosque, Cll 119 \# 7-75, Bogotá, Colombia. ${ }^{2}$ Department of Anesthesia and Perioperative Medicine, Intensive Care Medicine and Transplant Anesthesiologist, Hospital Universitario Fundación Santa Fe de Bogotá, Universidad de los Andes, Universidad el Bosque, Bogotá, Colombia.

Received: 30 June 2018 Accepted: 17 July 2018

Published online: 16 August 2018

References

1. Troianos CA, Hartman GS, Glas KE, et al. Guidelines for performing ultrasound guided vascular cannulation: recommendations of the American Society of Echocardiography and the Society of Cardiovascular Anesthesiologists. J Am Soc Echocardiogr. 2011;24:1291-318.

2. Rupp SM, Apfelbaum JL, Blitt C, et al. Practice guidelines for central venous access: a report by the American Society of Anesthesiologists Task Force on central venous access. American Society of Anesthesiologists Task Force on central venous access. Anesthesiology. 2012;116:539-73.

3. Amaya-Zuñiga WF, Raffan-Sanabria F, Niño de Mejía C, et al. Internal jugular vein cannulation: how much safety can we offer? Rev Colomb Anestesiol. 2015:43:76-86

4. Saugel B, Scheeren TWL, Teboul JL. Ultrasound-guided central venous catheter placement: a structured review and recommendationsfor clinical practice. Crit Care. 2017;21(1):225. 